\title{
BMJ Open Clinical characteristics of infective endocarditis in patients with antineutrophil cytoplasmic antibody or antiphospholipid antibody: a retrospective study in Shanghai
}

\author{
Zhuochao Zhou (D) , ${ }^{1}$ Junna Ye, ${ }^{1}$ Jialin Teng, ${ }^{1}$ Honglei Liu, ${ }^{1}$ Xiaobing Cheng, ${ }^{1}$ \\ Yue Sun, ${ }^{1}$ Yutong Su, ${ }^{1}$ Huihui Chi, ${ }^{1}$ Fan Wang (D) , ${ }^{1}$ Chengde Yang, ${ }^{1}$ Wei Jin ${ }^{2}$
}

To cite: Zhou Z, Ye J, Teng J, et al. Clinical characteristics of infective endocarditis in patients with antineutrophil cytoplasmic antibody or antiphospholipid antibody: a retrospective study in Shanghai. BMJ Open 2020;10:e031512. doi:10.1136/ bmjopen-2019-031512

- Prepublication history and additional material for this paper are available online. To view these files, please visit the journal online (http://dx.doi. org/10.1136/bmjopen-2019031512).

ZZ and JY contributed equally.

Received 18 May 2019

Revised 16 December 2019

Accepted 28 January 2020

Check for updates

(C) Author(s) (or their employer(s)) 2020. Re-use permitted under CC BY-NC. No commercial re-use. See rights and permissions. Published by BMJ.

${ }^{1}$ Department of Rheumatology and Immunology, Ruijin Hospital, Shanghai Jiao Tong University School of Medicine, Shanghai, China

${ }^{2}$ Department of Cardiology, Ruijin Hospital, Shanghai Jiao Tong University School of Medicine, Shanghai, China

Correspondence to Dr Chengde Yang; yangchengde@sina.com

Dr Wei Jin; jinwei_ivy@126.com

\section{ABSTRACT}

Objective This study aimed to characterise rheumatic manifestations and autoantibodies in 432 patients diagnosed with infective endocarditis (IE) in Shanghai. Design, setting and participants A retrospective study was conducted in Ruijin Hospital from 1997 to 2017. The clinical and laboratory characteristics of a total of 432 patients were analysed. In addition, the differences between patients with positive and negative antineutrophil cytoplasmic antibodies (ANCA) and antiphospholipid (aPL) antibodies as well as the survival rates of these patients were compared.

Results A total of 432 patients, including 278 male patients and 154 female patients, were included. The mean age of the patients was $46 \pm 16$ years. A total of 346 patients $(80 \%)$ had cardiac surgery, and 55 patients $(13 \%)$ died in the hospital. Among the IE patients, 104 were tested for either ANCA or aPL and were analysed in different groups. Twenty-one (24\%) positive ANCA patients were proteinase 3-ANCA positive. Compared with the ANCA-negative group, patients with positive ANCA had higher IgM $(p=0.048)$, lower haemoglobin $(p=0.001)$ and a higher likelihood of arthritis $(p=0.003)$. Twenty-one (40\%) aPL-positive patients had a higher erythrocyte sedimentation rate than was found in the aPL-negative group ( $p=0.003$ ). In addition, the survival rate of the ANCA-positive IE patients was lower $(p=0.032)$ than that of the ANCA-negative group, while there was no difference between patients with or without aPL antibodies $(p=0.728)$.

Conclusion This study supports the claim that rheumatic manifestations and autoantibodies are frequently present in patients with IE and might lead to early misdiagnosis. Physicians should pay more attention to the measurement of autoantibodies in these patients.

\section{INTRODUCTION}

Infective endocarditis (IE) is a microbial infection of the endocardium that usually involves the heart valves. Despite being relatively rare worldwide, IE has been reported in a steady incidence over the past three
Strengths and limitations of this study

- It was a retrospective study in a tertiary clinical centre spanned over 20 years.

- This study filled the gap in the study of the infective endocarditis (IE) population related to antineutrophil cytoplasmic antibodies (ANCA) or antiphospholipid (aPL) antibodies in China.

- The study analysed the clinical characteristics and survival rates of IE patients with and without ANCA or aPL antibodies.

- As it was a retrospective study, not all patients underwent analysis for ANCA and aPL autoantibodies.

- Deaths occurring at home or in other hospitals were not registered.

decades ranging from 2 to 6 per 100000 individuals in the general population per year and has a considerable associated mortality rate that varies from $10 \%$ to $30 \%{ }^{1}$ Predisposing factors for IE include conditions such as congenital heart diseases, rheumatic valve disease, artificial valves and other factors such as intravenous drug use (DU), dental procedures and haemodialysis. ${ }^{2}$ The diagnosis of IE is made based on symptoms, blood cultures, echocardiography and pathological biopsy from valve surgery. ${ }^{3}$ However, superantigens induced by some bacteria, such as Staphylococcus aureus, can stimulate an immune response, which could interfere with antibody production. ${ }^{4}$ Rheumatic manifestations, such as myalgia, arthralgia and arthritis are prevalent, occurring in nearly $40 \%$ of patients with IE at presentation of weeks to months before the diagnosis of IE. ${ }^{5}$ When classic IE manifestations are less evident, patients can be misdiagnosed as having a rheumatological disease, which might lead to delayed 
initiation of antibiotic treatment. Thus, the distinction between IE and rheumatic diseases is not always easy, and to improve the understanding concerning differences between these conditions is of great importance.

Many specific antibodies, such as antineutrophil cytoplasmic antibodies (ANCA) and anti-phospholipid (aPL) antibody, might be related to the pathophysiology of IE. ${ }^{6}$ Due to positive ANCA tests, IE has been reported to mimic ANCA-associated vasculitis (AAV). Patients with IE may present with multiple pulmonary nodules and glomerulonephritis, which mimics granulomatosis with polyangiitis. ${ }^{8}$ One study further underscored that ANCA might be associated with multiple valve involvement. ${ }^{6}$ In addition, infection-associated elevated aPL levels in patients with IE are related to endothelial cell activation, thrombin generation and impairment of fibrinolysis, which may contribute to the increased risk of major embolic events in these patients. ${ }^{7}$ Therefore, ANCA and aPL are not specific to IE patients.

The objective of this study was to compare the clinical characteristics and survival rates of IE patients with and without ANCA or aPL autoantibodies in a tertiary hospital in Shanghai, China from 1997 to 2017.

\section{METHODS \\ Patients}

This study was a retrospective study. We continuously included 432 patients hospitalised in Ruijin Hospital affiliated to Shanghai Jiao Tong University School of Medicine who were diagnosed with IE from 1997 to 2017 after ruling out 33 patients with primary rheumatic disease or in immunosuppression condition. The clinical and laboratory data of the patients were mainly obtained from the medical records system of Ruijin Hospital.

Only patients with definite IE determined by either the Duke criteria (up to 2000) or the modified Duke criteria (from 2000 onwards) were included. ${ }^{39}$ Duration of disease was defined as the period from the time when the patients presented with the first clinical feature to a definite IE diagnosis. In patients with long-term fever, the autoantibodies were tested to rule out rheumatic diseases. Cardiac surgery and death were recorded during the period of hospitalisation. The study followed the ethical standards for human experimentation established in the Declaration of Helsinki.

In this study, patients positive for either myeloperoxidase (MPO)-ANCA or proteinase 3 (PR3)-ANCA were defined as ANCA-positive patients. Patients with any positive test for anticardiolipin antibodies (ACL), lupus anticoagulant (LAC) or anti- $\beta 2$ glycoprotein I antibodies (aß2GPI) were defined as aPL-positive patients. Congestive heart failure was defined according to the New York Heart Association classification system. ${ }^{10}$ In-hospital mortality was defined as IE patients who died during hospitalisation. There was a 4-month observation period for patient mortality from the day of diagnosis. The survival rates of subgroups positive or negative for ANCA and aPL were then analysed.

\section{Laboratory features and echocardiography}

Levels of anti-PR3, anti-MPO and aPL antibodies in serum were measured by ELISA. The following laboratory data were recorded: white blood cell counts in blood, haemoglobin, platelets, C-reactive protein, erythrocyte sedimentation rate (ESR), lactate dehydrogenase, rheumatoid factor (RF), IgG, IgA, IgM, antinuclear antibody (ANA), ACL, LAC, aß2GPI antibodies, anti-PR3 antibodies (PR3-ANCA), anti-MPO antibodies (MPO-ANCA), haematuria and proteinuria. All IE patients underwent transthoracic or transoesophageal echocardiography. Furthermore, each patient underwent abdominal ultrasounds.

\section{Statistical analysis}

Data were analysed using the Statistical Package for the Social Sciences for Windows (V.23.0; SPSS, IBM). Statistical analyses were performed by t-test or $\chi^{2}$ test according to the type of data (continuous or categorical, respectively). Survival curves were obtained using the KaplanMeier method. Significance was obtained by a log-rank test. A p value of less than 0.05 was considered statistically significant.

\section{Patient and public involvement}

Patients were not informed by the development of the research question and outcome measures. Patients were not involved in the design, the recruitment to and conduct of the study as the study was retrospective. The results were not shared with study participants.

\section{RESULTS}

\section{Characteristics of patients}

As shown in table 1 , a total of 432 patients, including $278(64 \%)$ male patients and $154(36 \%)$ female patients (male:female ratio=1.8:1), were analysed. The mean age $\pm \mathrm{SD}$ of patients was $46 \pm 16$ years. The median duration of disease was $1.56(0.75,3)$ months. The mitral valve $(54 \%)$ was the most frequently involved valve followed by the aortic valve $(43 \%)$. The most common complication of IE patients was congestive heart failure in 141 patients $(33 \%)$. For embolic events discovered or presented in the hospital, brain $(14 \%)$ and spleen $(6 \%)$ infarction were most commonly seen. For predisposing factors, dialysis accounted for $1 \%$, dental procedures accounted for $1 \%$, and intravenous DU accounted for $0.5 \%$. In addition, $58(13 \%)$ patients had rheumatic valvulopathy. During hospitalisation, 346 patients $(80 \%)$ had cardiac surgery, and 55 patients $(13 \%)$ died. The pathogens associated with IE patients are listed in online supplementary table 1.

\section{Clinical manifestations and laboratory features}

Fever $(84 \%)$ and new heart murmur (79\%) were present in most patients with IE (online supplementary table 2 ). The most common rheumatic manifestations were 


\begin{tabular}{|c|c|}
\hline Item & $\mathbf{N}$ \\
\hline $\mathrm{N}$ & 432 \\
\hline Female/male & $\begin{array}{l}154(36) / 278 \\
(64)\end{array}$ \\
\hline Age (years, mean $\pm S D$ ) & $46 \pm 16$ \\
\hline Duration (months, median, IQR) & $1.56(0.75,3)$ \\
\hline \multicolumn{2}{|l|}{ Predisposing factors } \\
\hline Dialysis & $6(1)$ \\
\hline Dental procedure & $4(1)$ \\
\hline Intravenous drug use & $2(0.5)$ \\
\hline Congenital heart disease & $66(15)$ \\
\hline Rheumatic valvulopathy & $58(13)$ \\
\hline \multicolumn{2}{|l|}{ Complications } \\
\hline Congestive heart failure & $141(33)$ \\
\hline Hypertension & $82(19)$ \\
\hline Diabetes & $27(6)$ \\
\hline Cancer & $11(3)$ \\
\hline \multicolumn{2}{|l|}{ Embolic events } \\
\hline Brain & $67(14)$ \\
\hline Spleen & $26(6)$ \\
\hline Kidney & $8(2)$ \\
\hline Extremity & $8(2)$ \\
\hline Coronary & $4(1)$ \\
\hline Lung & $4(1)$ \\
\hline \multicolumn{2}{|l|}{ Infected valve } \\
\hline Prosthetic valve & $42(10)$ \\
\hline \multicolumn{2}{|l|}{ Native valve } \\
\hline Mitral valve & $234(54)$ \\
\hline Aortic valve & $185(43)$ \\
\hline Tricuspid valve & $17(4)$ \\
\hline Pulmonary valve & $10(2)$ \\
\hline \multicolumn{2}{|l|}{ Outcome } \\
\hline Cardiac surgery & $346(80)$ \\
\hline Relapse & $13(3)$ \\
\hline In-hospital mortality & $55(13)$ \\
\hline
\end{tabular}

$\mathrm{IE}$, infective endocarditis.

arthritis (15\%) and myalgia (6\%). In addition, a few patients had non-specific manifestations such as hepatomegaly $(4 \%)$, splenomegaly $(23 \%)$ and fatigue $(20 \%)$. Eighteen patients had Janeway lesions (4\%), 11 patients had Osler nodes $(3 \%)$ and five patients had Roth spot $(1 \%)$.

Blood cultures were positive in $50 \%$ of IE patients. Echocardiographic data showed that 266/432 (62\%) patients had IE-specific characteristics such as vegetation, abscess, pseudoaneurysm, intracardiac valvular perforation and abnormal activity around the site of the
Table 2 Antibodies in 38 patients tested for both ANCA and $\mathrm{aPL}$

\begin{tabular}{cl}
\hline & $\mathbf{N}$ \\
\hline aPL-positive & 15 \\
ACL-positive & 1 (IgM ACL) \\
aß2GP1-positive & 1 \\
LAC-positive & 10 \\
ACL+LAC & $3(2$ IgM ACL +1 lgA ACL) \\
ANCA-positive & 9 \\
Both aPL- and ANCA- & $3(L A C$, LAC + IgM ACL, IgM \\
positive & ACL) \\
\hline
\end{tabular}

ACL, anticardiolipin; a $32 \mathrm{GPI}$, anti- $\beta 2$ glycoprotein I; ANCA, antineutrophil cytoplasmic antibody; aPL, anti-phospholipid; LAC, lupus anticoagulant.

prosthetic valve. Regarding immunologic features, 89 patients were tested for ANCA, 21 (24\%) of these were ANCA-positive, and all of these were PR3-ANCA positive. Fifty-three patients were tested for aPL, and 21 were positive $(45 \%)$. One patient had positive IgG aPL (2\%), 4 patients had IgM aPL (8\%), 1 patient had IgA aPL (2\%), 2 patients had aß2GPI (4\%) and 17 patients had LAC (32\%). Eight (13\%) patients were positive for ANA, and one was positive for extractable nuclear antigen (ENA) $(2 \%)$. Proteinuria $(109 / 432,25 \%)$ and haematuria $(181 / 432,42 \%)$ were most commonly observed. Of 38 IE patients tested for both aPL and ANCA, 15 were aPL-positive, 9 were ANCA-positive and 3 were positive for both antibodies (table 2). The clinical features and laboratory features of patients tested for antibodies or not are presented in online supplementary table 3. Arthritis was more common in patients tested for antibodies than in those who were not, indicating the relevance between autoantibodies and rheumatic features.

\section{Comparison of patients with positive and negative ANCA or APL}

Of 432 IE patients, the 104 who were tested for either ANCA or aPL were analysed. Finally, 89 patients were classified as 'ANCA-positive IE' or 'ANCA-negative IE', and 53 patients were classified as 'aPL-positive IE' or 'aPL-negative IE' according to their antibodies test (online supplementary figure 1). Thirty-eight patients were tested for both ANCA and aPL antibodies, and their characteristics were analysed. Of 89 patients who were tested for ANCA, 21 (24\%) were ANCA-positive, and $68(76 \%)$ were ANCA-negative. Arthritis $(\mathrm{p}=0.003)$ was more frequent in ANCA-positive patients than in ANCA-negative patients, while more cardiac surgery was done in ANCA-negative patients $(\mathrm{p}=0.002)$ (table 3$)$. Other clinical manifestations such as fever, fatigue, embolic events, myalgia, splenomegaly, heart murmur and weight loss were not significantly different between the two groups $(\mathrm{p}>0.05)$. Compared with ANCA-negative patients, in ANCA-positive patients, IgM ( $\mathrm{p}=0.048)$ was 
Table 3 Clinical features of patients with positive or negative ANCA and aPL

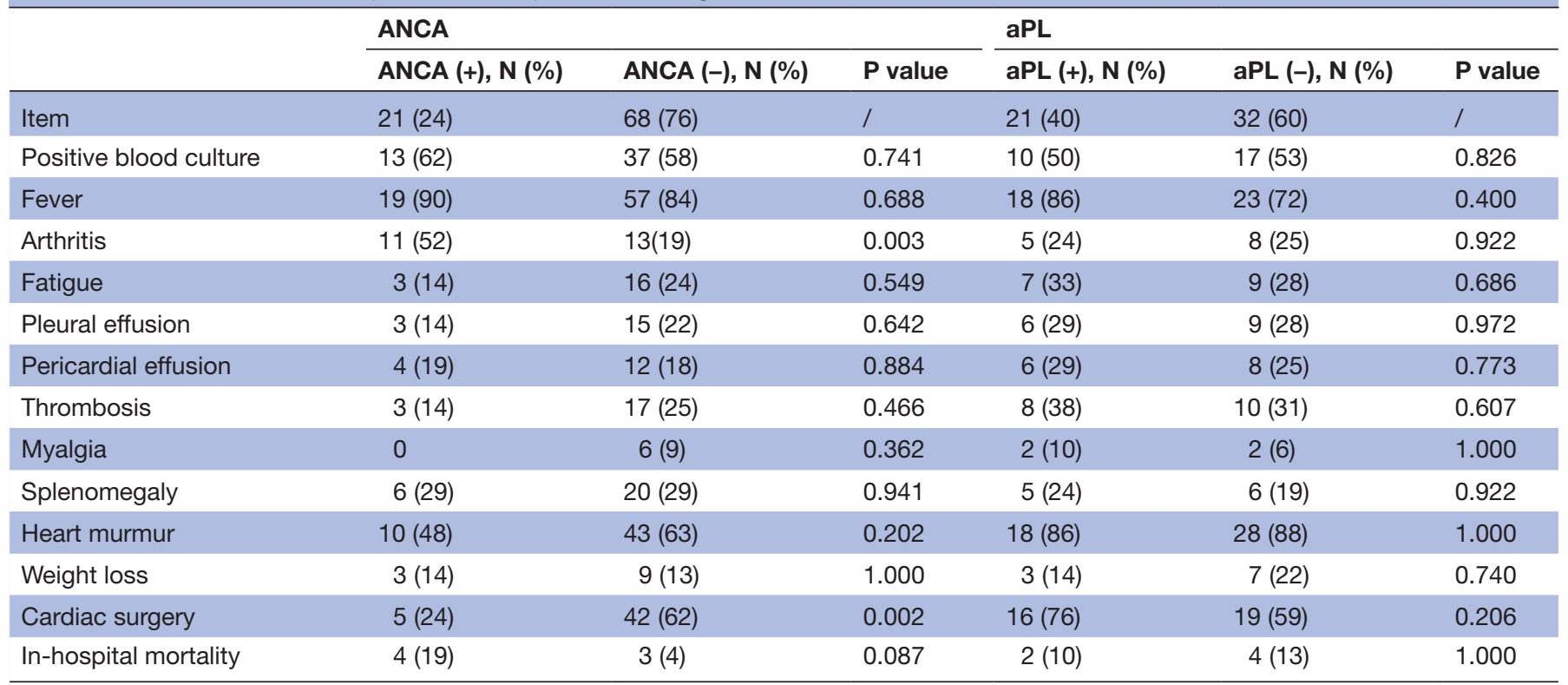

ANCA, antineutrophil cytoplasmic antibody; aPL, anti-phospholipid.

higher, $\mathrm{Hb}(\mathrm{p}=0.001)$ was significantly lower and elevated $\mathrm{RF}(\mathrm{p}=0.053)$ seemed to be more common (table 4$)$. Of 53 patients who were tested for aPL, 21 (40\%) were aPLpositive, and the remaining $32(60 \%)$ were aPL-negative. ESR was significantly higher in patients who were positive for aPL than in those who were negative $(p=0.003)$ (table 4).

\section{Survival rate}

Overall, 9 patients tested for aPL or ANCA antibodies died in the hospital. Among these patients, 4 were ANCApositive, and 2 patients were aPL-positive. One patient died of renal failure, four of acute heart failure, two of septic shock and two of stroke. The survival rate was significantly lower in ANCA-positive IE patients ( $\mathrm{p}=0.032)$, but there was

Table 4 Laboratory features of patients positive or negative for ANCA and aPL

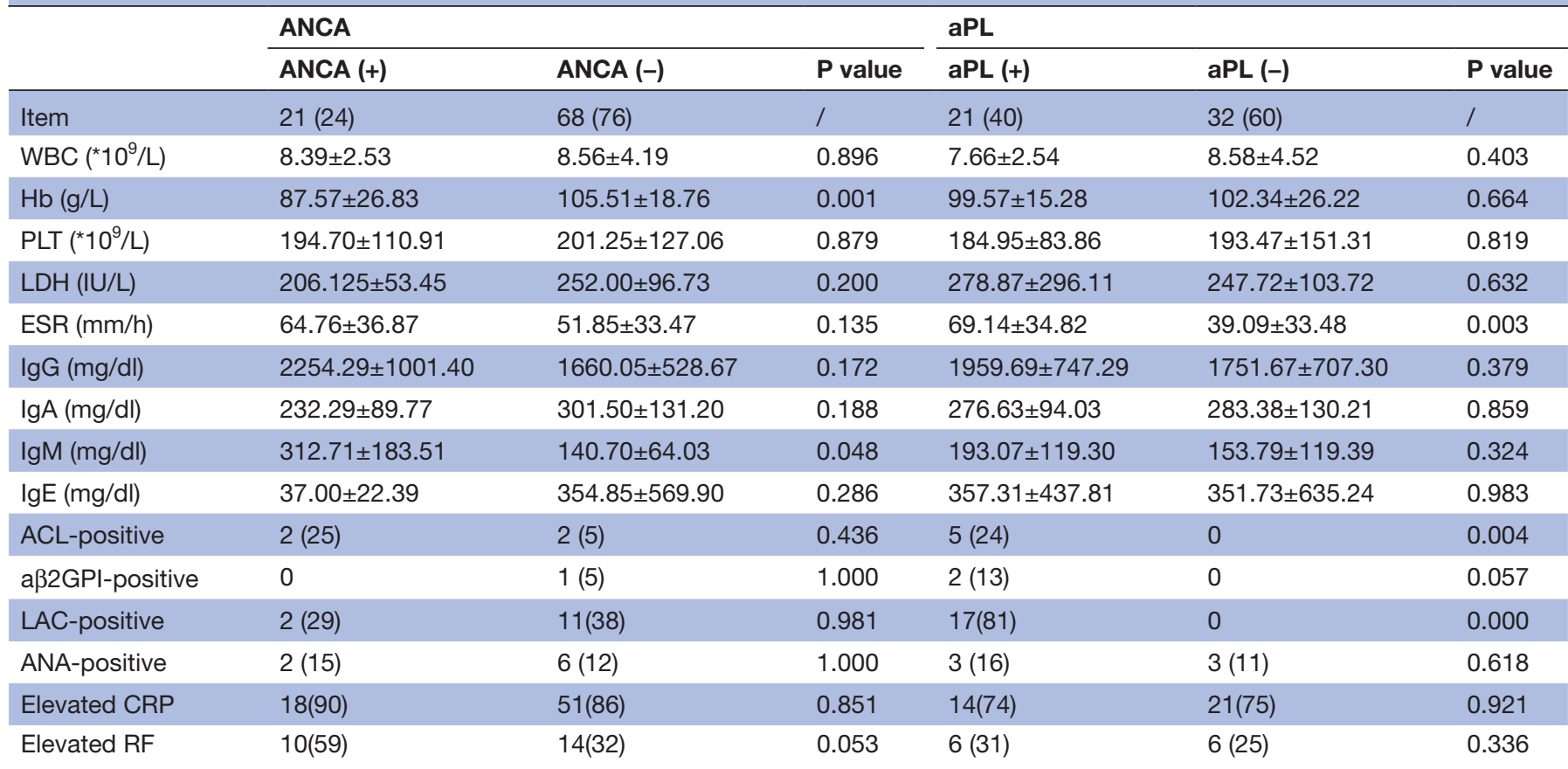

ACL, anticardiolipin; aß2GPI, anti- 32 glycoprotein I; ANA, antinuclear antibody; ANCA, antineutrophil cytoplasmic antibody; aPL, antiphospholipid; CRP, C-reactive protein; ESR, erythrocyte sedimentation rate; $\mathrm{Hb}$, haemoglobin; LAC, lupus anticoagulant; $\mathrm{LDH}$, serum lactate dehydrogenase; PLT, platelets; RF, rheumatoid factor; WBC, white blood cell. 
A
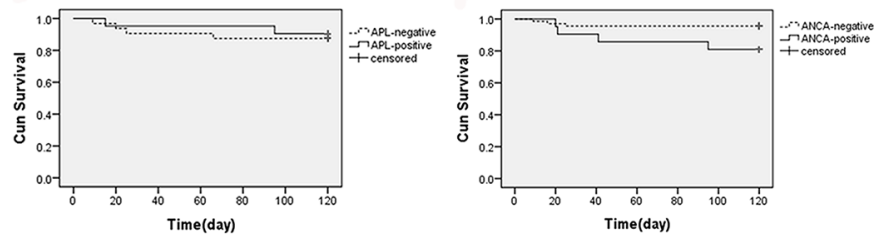

Figure 1 Kaplan-Meier survival curves. (a) Kaplan-Meier survival curves of patients who were tested for antiphospholipid (aPL). (B) Kaplan-Meier survival curves of patients who were tested for antineutrophil cytoplasmic antibody (ANCA).

no significant difference between the aPL groups $(\mathrm{p}=0.728)$ (figure 1).

\section{DISCUSSION}

Although it has been reported about different aspects of $\mathrm{IE}^{11-13}$ the analysis of the clinical and immunologic features of Chinese IE patients is still limited. The clinical presentations and laboratory findings of IE are similar to those of many rheumatic diseases, making it difficult to diagnose. Thus, it is pivotal to analyse the rheumatic manifestations of IE to help physicians differentiate it when encountering these clinical scenarios. In this study, we analysed the clinical and laboratory features of $432 \mathrm{IE}$ patients in Ruijin Hospital and compared the characteristics of 104 patients tested for ANCA or aPL during the past two decades. The mean age of the IE patients was 46 years. In many studies of IE patients, the mean age was higher than that in our study, while other IE patients were similar to ours. ${ }^{2}{ }^{14} 15$ In addition, elderly people were more likely to have valve replacement surgery because of degenerative valve disease. With regard to blood culture, it has been reported that blood culturenegative IE (BCNIE) may occur in up to $31 \%$ of all cases of IE and often poses considerable diagnostic and therapeutic dilemmas. ${ }^{3}$ BCNIE most commonly arises as a consequence of previous antibiotic administration. As a tertiary hospital, before hospitalisation in Ruijin Hospital, our patients had been treated with antibiotics empirically in other hospitals with uncertain diagnoses. As a result, only approximately $50 \%$ of the patients had a positive blood culture. Patients who were culture-negative were diagnosed based on other criteria such as echocardiography findings and a postoperative histological examination of vegetation. In addition, our study showed that ANCA-positive IE patients had higher IgM and lower $\mathrm{Hb}$ levels and were more likely to present with arthritis than was found in ANCA-negative IE patients. Moreover, aPLpositive IE patients had significantly higher ESR than was found in aPL-negative patients.

Positive ANCA and aPL were previously reported to be non-specific in patients with infection. ${ }^{6-8}$ The phenomenon of IE patients positive for ANCA has long been reported. A study from France reported that $8 \%$ of IE patients had PR3-ANCA or MPO-ANCA, associated with young age $(\mathrm{p}=0.022)$, echocardiographic vegetation $(\mathrm{p}=0.043)$ and elevated IgG levels $(\mathrm{p}=0.017) .{ }^{16}$ It has also been reported that most Gram-positive endocarditis can cause an anti-PR3 specificity. During delayed polymorphonuclear leucocyte apoptosis, PR3 contact with the immune system could possibly cause an increase in the production of anti-PR3 ANCA. ${ }^{17}$ It was interesting to find that among our IE patients tested for ANCA, all of them were PR3-ANCA-positive. One important presentation of AAV is glomerulonephritis. However, IE-associated glomerular nephritis often presents with acute kidney injury. ${ }^{18}$ The most common biopsy finding was necrotising and crescentic glomerular nephritis (53\%), followed by endocapillary proliferative glomerular nephritis $(37 \%){ }^{18}$ In our study, among IE patients, those with proteinuria and haematuria accounted for $25 \%$ and $42 \%$, respectively. However, the kidney biopsy was not routinely performed in these patients.

Apart from ANCA, aPL was also analysed in our study. In terms of the relationship between aPL and IE, it has been indicated that IgG aPL might be associated with $\mathrm{Q}$ fever endocarditis and aPL antibodies have previously been shown to be associated with thrombosis during acute $Q$ fever. ${ }^{19}{ }^{20}$ In our study, aPL-positive patients did not have positive serology of Coxiella. Furthermore, the presence of IgM aPL and $\mathrm{a}_{2}$ GPI was associated with embolic events, especially cerebral events, which could contribute to assessing the embolic risk of IE. ${ }^{21}$ In our study, there was no difference in thrombosis between aPL-positive patients and aPL-negative patients. APL-positive IE patients had higher ESR, and LAC was the most commonly positive type of aPL in IE patients. This might indicate that aPL was related to an inflammatory status.

In terms of the survival rate found in our study, it was significantly lower in the ANCA-positive IE group than in the ANCA-negative group. ANCA-positive patients were more likely to be mistaken for AAV, leading to a delay in diagnosis. Thus, these antibodies may be associated with a poor prognosis, which might be the reason for a higher mortality. Previously, we reported that the survival rate was significantly lower in ANCA-positive IE patients than in ANCA-negative group. ${ }^{13}$ However, there was no difference in the survival rate between the aPL groups in our study.

There were several limitations of this study. First, it was retrospective. The measurement of autoantibodies was not conducted in all IE patients when they were diagnosed, with only 89 undergoing ANCA and 53 aPL testing, and only 38 patients were tested for both ANCA and aPL antibodies. Second, the duration of the study spanned over 20 years, and some patients were lost to follow-up. Third, although there was a 4-month observation period for patients' mortality from the day of diagnosis during hospitalisation, we did not register deaths occurring in other hospitals or patients who died at home. 


\section{CONCLUSIONS}

This study supports the hypothesis that rheumatic complications are frequent in patients with IE. For rheumatologists, it is indispensable to routinely exclude IE in patients with rheumatic features such as arthritis, splenomegaly, myalgia, proteinuria and haematuria. For cardiologists, more attention should be paid to the measurement of autoantibodies in IE patients as this might contribute to the evaluation of prognosis.

Acknowledgements We would like to thank the patients and patient advisors for their participation in this study. We also thank Dr Shuzhen Xiao for her critical reading of this manuscript.

Contributors JT, HL, XC, HC and FW collected the clinical data. YSu and YSun analysed the data. ZZ and JY wrote the manuscript. WJ and CY designed the study and critically read the manuscript.

Funding This work is supported by the National Natural Science Foundation of China $(81801592,81370397,81670266)$, Science and Technology Commission of Shanghai Municipality (17140902500), Shanghai Sailing Program (18YF1414100), Shanghai Jiao Tong University Interdisciplinary Research Project (YG2016QN60), Excellent Youth B Project (GCQN-2017-B05) and Innovative research team of highlevel local universities in Shanghai.

Competing interests None declared.

Patient consent for publication Obtained.

Provenance and peer review Not commissioned; externally peer reviewed.

Data availability statement All data relevant to the study are included in the article or uploaded as supplementary information.

Open access This is an open access article distributed in accordance with the Creative Commons Attribution Non Commercial (CC BY-NC 4.0) license, which permits others to distribute, remix, adapt, build upon this work non-commercially, and license their derivative works on different terms, provided the original work is properly cited, appropriate credit is given, any changes made indicated, and the use is non-commercial. See: http://creativecommons.org/licenses/by-nc/4.0/.

ORCID iDs

Zhuochao Zhou http://orcid.org/0000-0003-1109-5781

Fan Wang http://orcid.org/0000-0001-6585-9711

\section{REFERENCES}

1 Fedeli U, Schievano E, Buonfrate D, et al. Increasing incidence and mortality of infective endocarditis: a population-based study through a record-linkage system. BMC Infect Dis 2011;11:48.

2 Monteiro TS, Correia MG, Golebiovski WF, et al. Asymptomatic and symptomatic embolic events in infective endocarditis: associated factors and clinical impact. Braz J Infect Dis 2017;21:240-7.

3 Habib G, Lancellotti P, Antunes MJ, et al. 2015 ESC guidelines for the management of infective endocarditis: the task force for the management of infective endocarditis of the European Society of cardiology (ESC). endorsed by: European association for CardioThoracic surgery (EACTS), the European association of nuclear medicine (EANM). Eur Heart $J$ 2015;36:3075-128.

4 Spaulding AR, Salgado-Pabon W, Kohler PL, et al. Staphylococcal and streptococcal superantigen exotoxins. Clin Microbiol Rev 2013;26:422-47.

5 Misra DP, Chowdury AC, Edavalath S, et al. Endocarditis: the great mimic of rheumatic diseases. Trop Doct 2016;46:180-6.

6 Langlois V, Lesourd A, Girszyn N, et al. Antineutrophil cytoplasmic antibodies associated with infective endocarditis. Medicine 2016;95:e2564.

7 Kupferwasser LI, Hafner G, Mohr-Kahaly S, et al. The presence of infection-related antiphospholipid antibodies in infective endocarditis determines a major risk factor for embolic events. J Am Coll Cardiol 1999;33:1365-71.

8 Peng H, Chen W-fang, Wu C, et al. Culture-negative subacute bacterial endocarditis masquerades as granulomatosis with polyangiitis (Wegener's granulomatosis) involving both the kidney and lung. BMC Nephrol 2012;13:174.

9 Durack DT, Lukes AS, Bright DK, et al. New criteria for diagnosis of infective endocarditis: utilization of specific echocardiographic findings. Am J Med 1994;96:200-9.

10 Yancy CW, Jessup M, Bozkurt B, et al. 2013 ACCF/AHA guideline for the management of heart failure: a report of the American College of cardiology Foundation/American heart association Task force on practice guidelines. J Am Coll Cardiol 2013;62:e147-239.

11 El-Chakhtoura N, Yasmin M, Kanj SS, et al. A 27 -year experience with infective endocarditis in Lebanon. $J$ Infect Public Health 2017;10:734-9.

12 Ferraris L, Milazzo L, Ricaboni D, et al. Profile of infective endocarditis observed from 2003 - 2010 in a single center in Italy. BMC Infect Dis 2013;13:545.

13 Ying C-M, Yao D-T, Ding H-H, et al. Infective endocarditis with antineutrophil cytoplasmic antibody: report of 13 cases and literature review. PLoS One 2014;9:e89777.

14 Şimșek-Yavuz S, Şensoy A, Kașıkçıoğlu H, et al. Infective endocarditis in turkey: aetiology, clinical features, and analysis of risk factors for mortality in 325 cases. Int $J$ Infect Dis 2015;30:106-14.

15 Ghosh S, Sahoo R, Nath RK, et al. A study of clinical, microbiological, and echocardiographic profile of patients of infective endocarditis. Int Sch Res Notices 2014;2014:340601

16 Mahr A, Batteux F, Tubiana S, et al. Brief report: prevalence of antineutrophil cytoplasmic antibodies in infective endocarditis. Arthritis Rheumatol 2014;66:1672-7.

17 Aslangul E, Goulvestre C, Mallat Z, et al. Human Bartonella Infective Endocarditis is Associated with High Frequency of Antiproteinase 3 Antibodies. J Rheumatol 2014;41:408-10.

18 Boils CL, Nasr SH, Walker PD, et al. Update on endocarditisassociated glomerulonephritis. Kidney Int 2015;87:1241-9.

19 Million M, Walter G, Bardin N, et al. Immunoglobulin G anticardiolipin antibodies and progression to $Q$ fever endocarditis. Clin Infect Dis 2013;57:57-64

20 Million M, Bardin N, Bessis S, et al. Thrombosis and antiphospholipid antibody syndrome during acute $Q$ fever: a cross-sectional study. Medicine 2017;96:e7578.

21 Selton-Suty C, Maigrat C-H, Devignes J, et al. Possible relationship between antiphospholipid antibodies and embolic events in infective endocarditis. Heart 2018;104:509-16. 